\title{
Verifying the low frequency pulsar polarimetry of the MWA
}

\author{
Steven E. Tremblay ${ }^{1,2}$, Mengyao Xue ${ }^{1,2}$, N. D. Ramesh Bhat ${ }^{1,2}$ \\ and Caterina Tiburzi ${ }^{3,4}$ \\ ${ }^{1}$ International Centre for Radio Astronomy Research (ICRAR), Curtin University, \\ Bentley, WA 6102, Australia \\ email: steven.tremblay@curtin.edu.au \\ ${ }^{2}$ ARC Centre of Excellence for All-sky Astrophysics (CAASTRO) \\ ${ }^{3}$ Max-Planck-Institut für Radioastronomie, Auf dem Hügel 69, 53121 Bonn, Germany \\ ${ }^{4}$ Universität Bielefeld, Fakultät für Physik, Universitätsstr. 25, D-33615 Bielefeld, Germany
}

\begin{abstract}
Studying the polarised properties of pulsars has a rich history giving unique geometric information about pulsars as well as testing the theories of pulsar emission physics. Performing such studies with the MWA has the attraction that the percentage of linear polarisation of many pulsars increases as the observing frequency decreases. Here we discuss the strategies being employed to verify the polarimetric response of the MWA's high time resolution data.
\end{abstract}

Keywords. pulsars: general, instrumentation: interferometers, polarization

\section{Introduction}

Polarimetric profiles of pulsars currently provide some of the best information available for understanding the geometry and emission from these compelling astrophysical sources.

The Murchison Widefield Array (MWA; Tingay et al. 2013) is an SKA-Low precursor located in Western Australia. A high time resolution system was implemented a couple of years ago (VCS; Tremblay et al. 2015) and it has since been performing pulsar research (e.g. Bhat et al. 2016, McSweeney et al. 2017). Up until now, however, the MWA hasn't published polarimetric analysis on these data since that mode has yet to be verified.

As an aperture array doesn't mechanically point, the raw polarimetric response to a given source on the sky is extraordinarily dependant to the position of that source. This makes the polarimetric calibration, an already complicated exercise, even more important for this type of array. To further complicate the issue, there is a dearth of well studied, low-frequency polarimetric profiles to compare the instrument against.

\section{Methodology}

In order to verify the polarimetric response of the MWA we have chosen to adopt a 'two-pronged' strategy. One can think of these broadly as: check for internal consistency, and compare with external data.

Firstly, we want to investigate the polarimetric response of the array as a function of pointing. Since the MWA is an aperture array with no moving parts, pointing is a purely electronic and software based process. While the instrumentation based delays can be fully known for a given observation, the ionosphere inevitably introduces its own tilebased delays which will both misdirect and distort our coherent beam if left uncorrected. To solve for these, as well as any instrumental delays not being properly modelled, we generate image based complex gain solutions for each tile. Doing so, however, means 


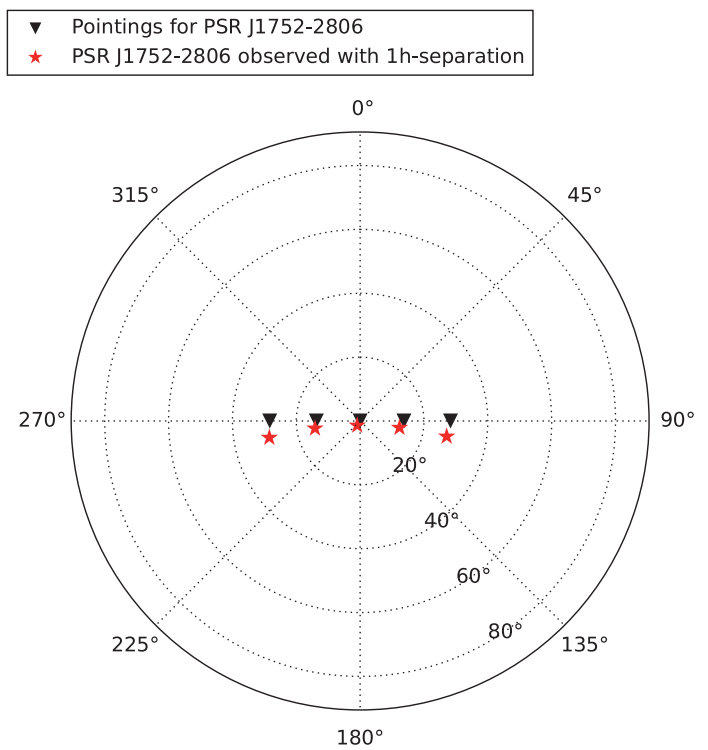

Figure 1. An example of using one of the bright pulsars that passes through Zenith at the MWA (PSR J1752-2806) across five subsequent pointings spaced one hour apart. Note how this not only samples our understanding of the instrument across a 60 degree span, but also the separation between the pulsar's position (stars) and the quantised pointing locations (inverted triangles).

that we're also dependant on our understanding of the response of an individual tile across the sky, as this is folded into the calibration process. Any errors in either the instrumental delays or the tile beam are almost certain to occur in a pointing dependant fashion. To test whether or not we observe such features we will observe two pulsars (PSRs J0752-2822 and J1742-2806) that pass through Zenith, each at a variety of hour angles (Fig. 1), and compare the Stokes parameters $(I, Q, U, V)$ as well as the polarisation angle curves for variation. This work will be presented in Xue et al. (in prep.).

Secondly, for a subset of pulsars we want to compare the polarised profiles and position angle sweeps with matched frequency data from another instrument. To do so, we're collaborating with the German long wavelength consortium (GLOW), who operate the German LOFAR stations (van Haarlem et al. 2013). We have selected a sample $(\sim 6)$ of commonly observable pulsars which span a wide range of periods and DMs as well as having unique polarimetric properties. This will allow each instrument to gain confidence in the polarimetric profile produced. This aspect of the project will be reported in a separate paper (Tremblay et al. in prep.).

\section{References}

Bhat, N. D. R.., Ord, S. M., Tremblay, S. E., McSweeney, S. J., \& Tingay, S. J. 2016, ApJ, 818, 86

McSweeney, S. J., Bhat, N. D. R.., Tremblay, S. E., Deshpande, A. A., \& Ord, S. M. 2017, ApJ, 836,224

Tingay, S. J., Goeke, R., Bowman, J. D., et al. 2013, PASA, 30, e007

Tremblay, S. E., Ord, S. M., Bhat, N. D. R., et al. 2015, PASA, 32, e005

van Haarlem, M. P., Wise, M. W., Gunst, A. W., et al. 2013, A\&̊A, 556, A2 